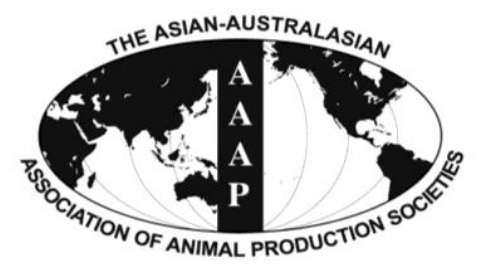

Asian-Aust. J. Anim. Sci.

Vol. 25, No. 7 : 1038 - 1044

July 2012

www.ajas.info

http://dx.doi.org/10.5713/ajas.2011.11324

\title{
Cytokines Expression and Nitric Oxide Production under Induced Infection to Salmonella Typhimurium in Chicken Lines Divergently Selected for Cutaneous Hypersensitivity
}

\author{
Rani Singh $^{1}$, Preeti Jain ${ }^{1}$, N. K. Pandey ${ }^{1}$, V. K. Saxena ${ }^{1}$, M. Saxena ${ }^{1}$, K. B. Singh ${ }^{2}$, \\ K. A. Ahmed ${ }^{1}$ and R. P. Singh ${ }^{1,3}, *$ \\ ${ }^{1}$ Disease Genetics and Biotechnology Laboratory, Central Avian Research Institute, Izatnagar, Bareilly, India \\ ${ }^{2}$ Department of Animal Science, M. J. P. Rohilkhand University, Bareilly, India
}

\begin{abstract}
In the present study, the impact of Salmonella Typhimurium on cell-mediated immunity (CMI) was investigated in 5 week-old immuno divergent broiler lines selected for the high and low response to phytohemagglutinin-P. The immune response was assessed in peripheral-blood mononuclear cells (PBMCs) induced with Salmonella Typhimurium at different time intervals (0 h, $0.5 \mathrm{~h}$, $2 \mathrm{~h}, 4 \mathrm{~h}, 6 \mathrm{~h}, 12 \mathrm{~h}$ and $24 \mathrm{~h}$ ). The differential mRNA expression patterns of IFN- $\gamma$, IL-2 and iNOS were evaluated by quantitative real time PCR. In-vitro production of nitric oxide (NO) was also estimated in the culture supernatant and correlated with iNOS mRNA expression. Present study showed higher production of NO in the high cell-mediated line (HCMI) as compared to the low cell-mediated line (LCMI) upon stimulation with Salmonella Typhimurium. Correspondingly, higher mRNA expression of iNOS and IFN- $\gamma$ were observed in high response birds (HCMI); but IL-2 was down regulated in this line compared to the low response birds (LCMI). Significantly $(\mathrm{p}<0.05)$ higher expression of iNOS, IFN- $\gamma$ and higher production of NO in high line indicated that the selection for PHA-P response might be employed for increasing the immune competence against Salmonella Typhimurium in chicken flocks. (Key Words: Phytohaemagglutinin-P (PHA-P), Nitric Oxide (NO), Peripheral Blood Mononuclear Cells (PBMCs))
\end{abstract}

\section{INTRODUCTION}

Salmonella carrier state in poultry has serious consequences to public health. Consumption of contaminated meat, eggs, and derived products may trigger food poisoning, which is particularly dangerous for human. Salmonella is also causing infection in chickens. In live birds, immune response generated against the infection which is a combination of three factors; antibody response (humoral immunity), $\mathrm{T}$ cell-mediated immunity and phagocytosis. It is reported that the degree of immune responses varied bird to bird, and believed that these facets of the immune system are influenced by individual's genome (Cheng and Lamont, 1988; Sarker et al., 2000) thus,

\footnotetext{
* Corresponding Author: Ram Pratap Singh. Tel: +917599061735, Fax: +91-4222657088, E-mail: rampratapsingh81@ gmail.com

${ }_{3}^{3}$ Avian Physiology and Genetics Division, Salim Ali Centre for Ornithology and Natural History, Anaikatty 641 108, Coimbatore, India.

Submitted Sept. 9, 2011; Accepted Dec. 5, 2011; Revised Dec. 28, 2011
}

indicating varying degree of resistance among the individuals. Recent advances in genetics and immunology have identified several factors that influence genetic resistance in chickens (Sadeyen et al., 2006; Wigley et al., 2006). Among these factors, cytokines are considered as useful bio markers for poultry breeders to develop new immuno-competent lines of birds resistant to salmonella infection (Swaggerty et al., 2004; Cheeseman et al., 2007; Van Hemert et al., 2007).

Cytokines are involved in regulation of both innate and adaptive immune responses. Delayed-type hypersensitivity reactions are mediated by a Th1 subset of CD4+ Th cells, which secrete IL-2, IFN- $\gamma$, TNF- $\alpha$ and GM-CSF cytokines, and are responsible for many cell-mediated functions. Cytokines from TH1 cells, particularly IFN- $\gamma$ and IL-2 mediate the differentiation of fully cytotoxic T-cells from CD8+ precursors. IFN $-\gamma$ is responsible for macrophage activation that results in increased NO production (Goldsby et al., 2003). NO, usually produced by activated mononuclear phagocytes, has antimicrobial and antiviral activities. It is an important mediator of immune and 
inflammatory responses, produced by macrophages through activation of inducible enzyme nitric oxide synthase (Djeraba et al., 2000). The iNOS found in the immune cells such as monocytes and macrophages are induced by intracellular pathogens, microbial products such as IFN- $\gamma$ to generate large quantity of NO (MacMicking et al., 1997). Production of NO by activated monocytes/macrophages is an important innate immune response, critical for bactericidal activity. The induction of iNOS in chicken macrophages has been shown to be associated with different genetic backgrounds (Hussain and Qureshi, 1998).

Indirect selection based on immune competent traits has been adjudged as the long-term strategy for developing disease resistant stocks (Lamont, 1998; Sundaresan et al., 2005). Earlier studies found that indirect selection based on PHA-P response resulted in divergent effect on Th1 cell activity, leading to more resistant birds against Escherichia coli (Sundaresan et al., 2005). Thus, we hypothesized that the indirect selection for PHA-P response might lead to more resistant birds against Salmonella. To verify this, the mRNA expression of IFN- $\gamma$, IL- 2 and iNOS were estimated, and in addition in-vitro productions of NO were evaluated in cultured peripheral-blood mononuclear cells (PBMCs) isolated from divergent chicken lines (HCMI and LCMI; Sundaresan et al., 2005), post induction to Salmonella.

\section{MATERIALS AND METHODS}

"All the experiments were performed in accordance with the rules of the Animal Ethics and Monitoring Committee of the Institute".

\section{Bacterial strain}

Standard strain of Salmonella Typhimurium E 2391 was procured from National Salmonella Centre, Indian Veterinary Research Institute, Izatnagar.

\section{Genetic background of experimental birds}

In the present study, immuno-divergent broiler lines selected for high (HCMI line) and low (LCMI line) PHA-P responses for 5 consecutive generations were utilized (Sundaresan et al., 2005). Ten extreme responder birds each from the HCMI and LCMI line, with an average foot web index of $184.08 \%$ and $6.58 \%$ respectively, were selected and used for this study. These birds were certified as free of Salmonella antigen as well as antibodies by the Avian Medicine Section of the Institute.

\section{Differential expression of IFN- $\boldsymbol{\gamma}$, IL-2 and iNOS genes}

Peripheral blood mononuclear cells (PBMCs) culture: PBMCs were separated from the extreme responder birds of both HCMI and LCMI line at 5 wks age, using Histopaque1.077 (Sigma Diagnostics Inc., St. Louis, MO, USA) as described earlier (Sundaresan et al., 2005). PBMCs collected from individual birds were assessed for the viability and then pooled. Four aliquots per line containing $10^{6}$ cells per $\mathrm{ml}$ were made in the RPMI-1640 (without phenol red) mediums supplemented with $10 \%$ FBS, $2 \mathrm{mM}$ L-glutamine, $2 \mathrm{mM}$ L-argenine under $5 \% \mathrm{CO}_{2}$ tension in a humidified atmosphere. Each aliquot was plated in 24 well tissue culture plate $\left(10^{6}\right.$ cells per well) as triplicate for each time interval $(0 \mathrm{~h}, 0.5 \mathrm{~h}, 2 \mathrm{~h}, 4 \mathrm{~h}, 6 \mathrm{~h}, 12 \mathrm{~h}$, and $24 \mathrm{~h})$. In each triplicate, two wells were induced with $10^{6}$ cells of Salmonella Typhimurium. The remaining well was kept as uninduced control. After PBMCs culture, the adherent cells were harvested at different time points. Harvested cells were collected in $1.5 \mathrm{ml}$ plastic tubes and centrifuge at $5,000 \times \mathrm{g}$ for $1 \mathrm{~min}$. After centrifugation, the supernatant was collected in a fresh tube for NO estimation, and pellet was dissolved in denaturing solution for RNA isolation. The total RNA was extracted by 'RNAgents- Total RNA isolation system' (Promega, Madison, WI, USA) according to the manufacturer's instructions. The RNA integrity was checked by $1 \%$ denaturing agarose gel electrophoresis and concentration was measured through Nanodrop, and the ratio of $260 / 280$ was $\geq 2.0$ for all the samples. The possible traces of genomic DNA were removed by treating $5 \mu \mathrm{g}$ of each RNA sample with $5 \mathrm{U}$ of RNase-free DNase (Biogene, $\mathrm{CA}$, USA) on $37^{\circ} \mathrm{C}$ for $1 \mathrm{~h}$. The DNase was subsequently inactivated by incubation at $65^{\circ} \mathrm{C}$ for $10 \mathrm{~min}$. Each DNase treated total RNA sample $(5 \mu \mathrm{g})$ was reverse transcribed with suitable negative and positive controls using the 'Revert Aid First strand cDNA synthesis kit' (MBI Fermentas, Hanover, MD, USA) according to the manufacturer's instructions. The resultant cDNA was stored frozen at $-20^{\circ} \mathrm{C}$ until used. Negative controls were performed using all components except reverse transcriptase. Total RNA from chicken spleen was used in positive controls and for standardizing reaction conditions.

\section{Quantification of IFN- $\gamma$, IL-2and iNOS}

Expression of IFN- $\gamma$, IL-2 and iNOS mRNA was quantified by real-time PCR by using the Syber Green master mix in IQ5 cycler (Biorad, USA). The gene-specific primer pairs (Table 1) were used from the published reports (Sundaresan et al., 2005). An initial validation experiment was conducted to confirm the specificity of primers (data not shown). $\beta$-Actin was used as a house keeping gene. All PCR reactions were performed in optical 96-well reaction plates in duplicate. The amplification was carried out in 25 $\mu$ volume containing 1X QuantiTect Syber Green PCR master mix (SYBR Green 1 dye, ROX passive reference dye, Hot Star Taq DNA polymerase and dNTPs with dUTP in optimized buffer components; QIAGEN $\mathrm{GmBH}$, Germany), a $0.2 \mu \mathrm{M}$ concentration of each gene-specific 
Table 1. Primers used for real time PCR

\begin{tabular}{|c|c|c|c|c|c|}
\hline SL. No. & Gene & Primer sequence & $\operatorname{Tm}\left({ }^{\circ} \mathrm{C}\right)$ & Size (bp) & Accession no. \\
\hline 1 & IFN- $\gamma$ & $\begin{array}{l}\text { F- 5’ATGACTTGCCAGACTTACAACTTG 3' } \\
\text { R-5'TTAGCAATTGCATCTCCTCTGAGA } 3^{\prime}\end{array}$ & 52 & 495 & AJ634956 \\
\hline 2 & IL-2 & $\begin{array}{l}\text { F-5'ATGATGTGCAAAGTACTGATC3' } \\
\text { R-5'TTATTTTTGCAGATATCTCAC3' }\end{array}$ & 50 & 432 & AJ578467 \\
\hline 3 & iNOS & $\begin{array}{l}\text { F-5'AGGCCAAACATCCTGGAGGTC } 3 \\
\text { R-5'TCATAGAGACGCTGCTGCCAG } 3 \text {, }\end{array}$ & 52 & 371 & U46504 \\
\hline 4 & $\beta$-Actin & $\begin{array}{l}\text { F-5'CATCACCATTGGCAATGAGAGG 3', } \\
\text { R-5'GCAAGCAGGAGTACGATGAATC 3' }\end{array}$ & 60 & 353 & L08165 \\
\hline
\end{tabular}

primer, and $1 \mu \mathrm{l}$ of cDNA template. PCR cycling conditions were: initial denaturation of $95^{\circ} \mathrm{C}$ for $15 \mathrm{~min}$, followed by 45 cycles of denaturation $95^{\circ} \mathrm{C}$ for $30 \mathrm{~s}$; annealing (Table 1) for $30 \mathrm{~s}$ and extension $72^{\circ} \mathrm{C} ; 45 \mathrm{~s}$. For each gene of interest, negative and positive controls were included. Recombinant plasmids with insert of IFN- $\gamma$, IL-2 and iNOS were used as a positive control. Negative controls were samples in which cDNA was not added. For each sample, a dissociation curve was generated after completion of amplification and analyzed in comparison to negative and positive controls, to determine the specificity of PCR reaction. Moreover, few amplicons were sequenced to confirm the specificity of primers. Results are expressed in terms of the threshold cycle value $(\mathrm{Ct})$, the cycle at which the change in the reporter dye $(\mathrm{DRn})$ passes the significance threshold. To generate gene-specific standard curves, a ten-fold dilution series of plasmids containing each of the different genes were used. Regression analysis of the standard curve was used to calculate the slopes of the gene specific $\log 10$ dilution series. To convey the inverse relationship between starting template concentration and $\mathrm{Ct}$ value, results were expressed and analyzed as a $40-\mathrm{Ct}$ value. The corrected cytokine mRNA per sample was calculated using the following formula:

$$
\begin{aligned}
\text { Adjusted } \mathrm{Ct} \text { value }= & (\text { mean } 40-\mathrm{Cttarget})(\text { Slopetarget }) \\
& /(\beta \text {-actin df })(\text { Slope } \beta \text {-actin })
\end{aligned}
$$

Where, mean 40-Cttarget $=$ the triplicate mean of 40-Ct value; Slopetarget $=$ the slope from the standard curve regression equation for the target gene; $\beta$-actin $\mathrm{df}=$ the triplicate mean of $\beta$-actin/overall mean for all $\beta$-actin values within the experiment; and Slope $\beta$-actin = the slope from the standard curve regression equation for the $\beta$-actin gene.

\section{In vitro NO production assay}

Selected 10 extreme responders of the HCMI and LCMI lines were utilized for in vitro NO production assay. PBMCs were cultured separately for each sample, as mentioned earlier. The cell-free supernatant was harvested at different time intervals $(0 \mathrm{~h}, 0.5 \mathrm{~h}, 2 \mathrm{~h}, 4 \mathrm{~h}, 6 \mathrm{~h}, 12 \mathrm{~h}$, and $24 \mathrm{~h}$ ) and NO estimation was carried out as per the method of Sastry et al. (2002). Briefly, $100 \mu$ of sample or standard was placed in a test-tube to which $400 \mu \mathrm{l}$ of $0.55 \mathrm{M}$ carbonate buffer ( $\mathrm{pH} 9.0$ ), followed by a small amount of copper-cadmium alloy filings (approximately $100 \mathrm{mg}$ ) were added and incubated at $30^{\circ} \mathrm{C}$ for $1 \mathrm{~h}$ with gentle shaking. After $1 \mathrm{~h}, 100 \mu \mathrm{l}$ of $0.35 \mathrm{M}$ sodium hydroxide and $400 \mu \mathrm{l}$ of $0.12 \mathrm{M}$ Zinc sulphate was added and incubated for $10 \mathrm{~min}$ at room temperature. The tubes were centrifuged at $4,000 \times$ $\mathrm{g}$ for $10 \mathrm{~min}$ and the supernatant transferred to wells of microtiter plates in triplicate. Then, $75 \mu \mathrm{l}$ of $1 \%$ sulphanilamide and $75 \mu \mathrm{l}$ of $0.1 \% \mathrm{~N}$-naphthalene diamine were added with gentle mixing. After a10-min incubation, the absorbance was measured at $545 \mathrm{~nm}$ in an ELISA reader (Specta MAX; Molecular Devices, USA). Negative and positive controls were also loaded in parallel on the same plate RPMI 1640 mediums were taken as blank. Nitrite concentrations were determined using sodium nitrite as standard.

\section{Statistical analysis}

The relative amount of mRNA expression of each gene (ratio) and NO were analyzed using least-square analysis (Harvey, 1975), considering lines as main effect in a fixedeffect model.

\section{RESULTS}

The expression of IFN- $\gamma$, IL- 2 and iNOS mRNAs were noticed in the PBMCs of controls, as well as Salmonella Typhimurium infected PBMCs of divergent chicken lines selected for PHA-P responses. A rapid increase of IFN- $\gamma$ mRNA expression was observed in both lines after Salmonella Typimurium induction. The expression of IFN- $\gamma$ mRNA was significantly higher $(\mathrm{p}<0.05)$ at 0.5 to $6 \mathrm{~h}$ in HCMI as compared to LCMI line (Figure 1) whereas, in LCMI line, the mRNA expression of IFN- $\gamma$ was significantly higher $(\mathrm{p}<0.05)$ at later intervals $(12$ to $24 \mathrm{~h})$. IL-2 was significantly $(\mathrm{p}<0.05)$ down regulated in HCMI 


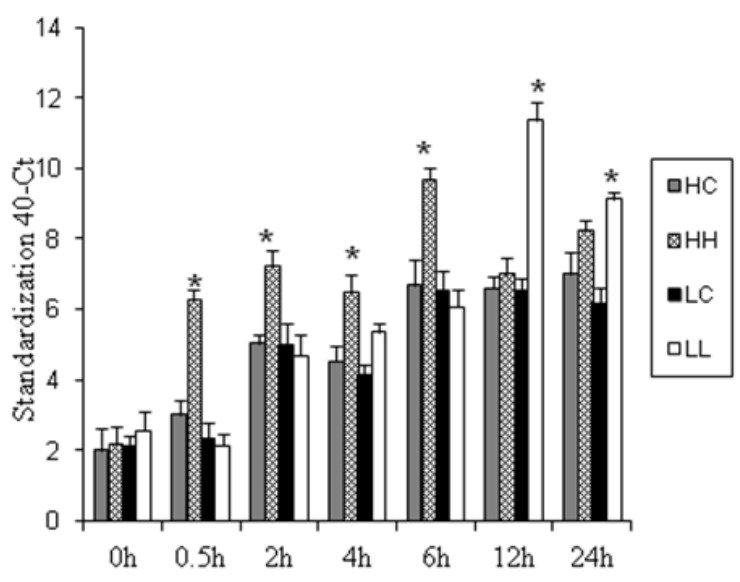

Figure 1. IFN-gamma mRNA expression in chicken peripheral blood mononuclear cells (PBMCs) of HCMI line and LCMI line with or without $S$. Typhimurium infection $(\mathrm{HC}=$ High line uninduced control; $\mathrm{HH}=$ High line induced with $S$. Typhimurium; LC $=$ Low line un-induced control; low line induced with $S$. Typhimurium).

line at all intervals studied. In contrast, significant $(\mathrm{p}<0.05)$ upregulation of IL-2 expression was noticed in LCMI line except 2 h (Figure 2).

iNOS was upregulated in both the lines at all the time points except $24 \mathrm{~h}$ after induction with Salmonella Typhimurium. The iNOS mRNA expression was higher $(\mathrm{p}<0.05)$ at $0.5 \mathrm{~h}, 2 \mathrm{~h}$ and $6 \mathrm{~h}$ time points in HCMI line as compared to LCMI line (Figure 3).

In-vitro NO production was observed in PBMCs cultured supernatant in control as well as Salmonella Typhimurium treated samples. A rapid peak of NO production was observed at $0.5 \mathrm{~h}$ in both the lines after that gradually decreased up to $6 \mathrm{~h}$. NO production was significantly $(\mathrm{p}<0.05)$ higher at $2 \mathrm{~h}$ and $4 \mathrm{~h}$ in HCMI line (Figure 4) as compared to LCMI line.

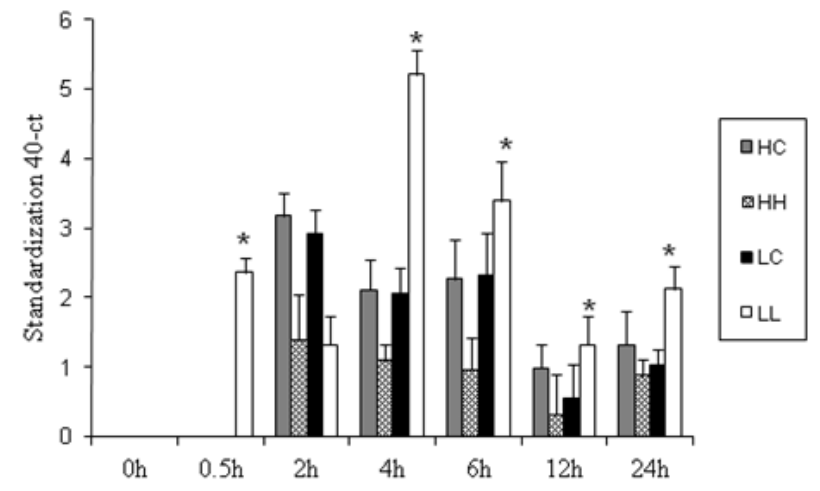

Figure 2. IL-2 mRNA expression in chicken peripheral blood mononuclear cells (PBMCs) of HCMI line and LCMI line with or without $S$. Typhimurium infection $(\mathrm{HC}=\mathrm{High}$ line un-induced control; $\mathrm{HH}=$ High line induced with $S$. Typhimurium; LC = Low line un-induced control; low line induced with $S$. Typhimurium).

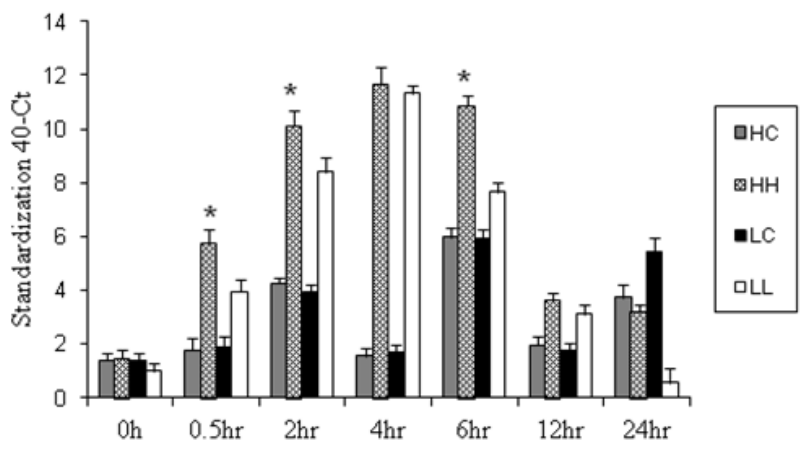

Figure 3. iNOS mRNA expression in chicken peripheral blood mononuclear cells (PBMCs) of HCMI line and LCMI line with or without $S$. Typhimurium infection $(\mathrm{HC}=$ High line un-induced control; $\mathrm{HH}=$ High line induced with $S$. Typhimurium; $\mathrm{LC}=$ Low line un-induced control; low line induced with $S$. Typhimurium).

\section{DISCUSSION}

Innate immunity plays an important role in regulation of Salmonella infections via modulating the acquired immune response. Earlier studies on Salmonella infections in chicken revealed the role of the humoral and cell-mediated immune response (Zhang-Barber, 1999; Berndt and Methner, 2001). Cell-mediated immunity is the most important in Salmonella infections because of antibodies, although abundantly produced locally, cannot access and act on these intracellular pathogens (Lillehoj et al., 2004). In the present study, PBMCs were selected for studying the genetic influence of IFN- $\gamma$, iNOS mRNA expression and production of nitric oxide. Because, PBMCs are easily accessible immune cells and can be harvested from breeder birds without sacrifice (Hangalapura et al., 2006).

IFN- $\gamma$ plays a vital role in macrophages activation and modulation of the immune system, in addition to an anti-

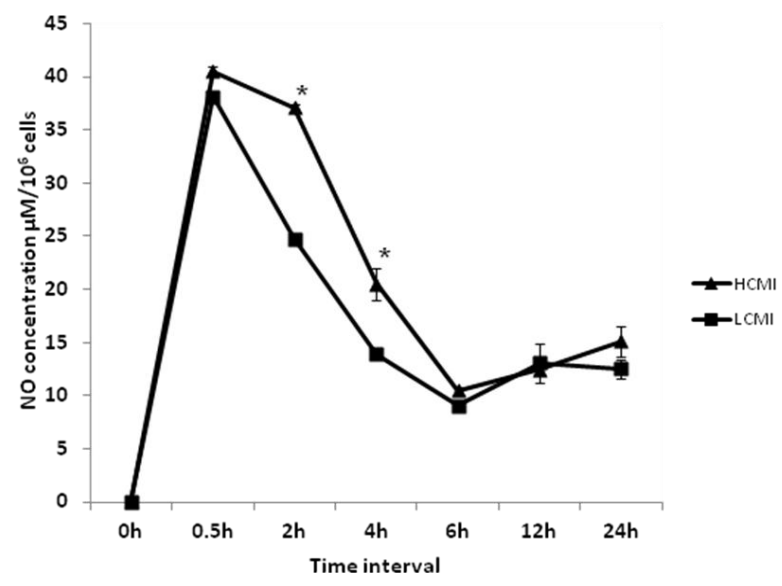

Figure 4. Nitric oxide production in chicken peripheral blood mononuclear cells (PBMCs) of HCMI line and LCMI line with or without $S$. Typhimurium infection $(\mathrm{HCMI}=$ High line; $\mathrm{LCMI}=$ Low line). 
viral activity. It is a major cytokine mediating resistance to many pathogens, including Salmonella (Sadeyen et al., 2004). INF- $\gamma$ is a major Th1 cytokine which plays an important role in protection against Salmonella infection in the avian host through the activation of macrophages to produce NO. A significantly low expression of IFN- $\gamma$ was observed in susceptible birds in comparison to resistant ones (Sadeyen et al., 2004). The present findings of significantly higher mRNA expression of IFN- $\gamma$ in HCMI line are in accordance with Sunderasan et al. (2005). The mRNA expression of was significantly low in LCMI line as compared to HCMI line. The Salmonella susceptible birds produced low IFN- $\gamma$ in comparison to resistant ones (Sadeyen et al., 2004). The present finding an earlier report suggested that IFN- $\gamma$ is an early expressing cytokine responsible for the immediate onset of immune reaction. Therefore, the results suggest that the higher level of IFN- $\gamma$ in HCMI line might be related to the genetic resistance. The reason for high level of IFN- $\gamma$ expression in HCMI line might be the higher frequency of $-318 \mathrm{GG}$ genotype in IFN- $\gamma$ promoter as compared to LCMI line where -318AA genotype was at a higher frequency (Sundaresan et al., 2005). The higher expression of IFN- $\gamma$ is also responsible for higher expression of iNOS in HCMI line to induce macrophages for a surge of NO. Comparatively, low level of IFN- $\gamma$ from $4 \mathrm{~h}$ onward indicated possible switchover to other cytokine cascades.

Chicken IL-2 exercises as an array of biological effects on many cells ( $\mathrm{T}$ lymphocytes, including NK cells, B cells, monocytes, and neutrophils) including the functional activation of cells of the innate immune response (Kogut et al., 2002). In the present study, the expression of IL-2 was down regulated in HCMI line as describer earlier by Kaiser et al. (2000). However, the LCMI line showed significantly higher levels of IL-2 expression. Similar results were observed in earlier studies conducted in these divergent lines (Sundaresan et al., 2005). The down regulation of IL-2 in HCMI line might be due to the higher levels of IFN- $\gamma$ (Imanshi, 2004; Sundaresan et al., 2005). However, further studies are required to find the exact reasons for the low expression of IL-2 in HCMI line.

The host immune response is modulated through the release of cellular components upon pathogenic exposure. A wide variety of cells is able to produce nitric oxide, which plays a key role in the innate response against infection. Enterobacteriaceae bacteria directly activate expression of iNOS and produced NO. NO is an important mediator of innate and acquired immunity (Lillehoj and $\mathrm{Li}, 2004$ ). In addition to direct microbicidal action, NO has immune regulatory effects relevant to the control of infections. In the present study, in vitro NO production was higher in HCMI line after induction with Salmonella Typhimurium. Djeraba et al. (2002) also reported that high immuno responsive birds showed higher NO production. We also found that divergent selection based on PHA-P response also showed significant differences in NO production between both lines. Present observations suggested a positive correlation between PHA-P response and NO production. Our previous studies showed that indirect selection based on PHA-P response resulted in divergent effect on Th1 cell activity, leading to enhance immune responsiveness to Escherichia coli (Sundaresan et al., 2005) and Newcastle disease virus (Ahmed et al., 2007). iNOS is responsible for the production of nitric oxide from macrophages. The iNOS enzymes are expressed only after activation by stimulants, such as endotoxins or cytokines. In chicken phagocytes, LPS and IFN- $\gamma$ are classic inducer of iNOS mRNA and nitric oxide production within hours of exposure. The peak of iNOS mRNA expression by chicken macrophages has been reported between 6 and $12 \mathrm{~h}$ after stimulation with LPS (Hussein and Qureshi, 1997). iNOS peak was observed at $4 \mathrm{~h}$ after induction with Salmonella Typhimurium in both lines. However, after Salmonella Typhimurium, expression of iNOS mRNA was higher at 0.5, 2 and $6 \mathrm{~h}$ in HCMI line as compare to LCMI line. The production of NO was higher at 2 and $4 \mathrm{~h}$ in HCMI line in PBMCs culture.

NO production in response to Salmonella Typhimurium was found correlated with the iNOS mRNA expression in PBMC culture, supporting the transcriptional regulation of the iNOS gene (Dil and Qureshi, 2002b). Hussain and Qureshi (1997) have reported that the concentration of iNOS mRNA was observed at $2 \mathrm{~h}$, whereas, the nitrite concentration was detected at $4 \mathrm{~h}$ after induction with LPS. Withanage et al. (2005) also found the NO production at $4 \mathrm{~h}$ after induction with live Salmonella and LPS. In present study, almost similar results were obtained. The times of $2 \mathrm{~h}$ have been lapsed between iNOS expression and formation of NO. It may be implied that a time of $2 \mathrm{~h}$ is required for the formation of NO and its release in the system since the formation of iNOS mRNA. The expression of iNOS mRNA was found higher in the HCMI against LCMI line. Present observations support our earlier observation of a positive correlation between PHA-P response and NO production (Sundaresan et al., 2005).

In Conclusion, the cell-mediated immunity is the only protective immune mechanism in the early phases in Salmonella infection. Moreover, it is effective in the Salmonella intracellular phase. The significantly higher mRNA expression of IFN- $\gamma$, iNOS and higher production of NO in HCMI line selected for a higher response to PHA-P, an indicator of the cell-mediated immune response, suggested a positive association between PHA-P response and immune responsiveness to Salmonella Typhimurium. Furthermore, PHA-P response might be exploited as a 
selection criterion to develop chicken lines having better immunocompetence against Salmonella.

\section{ACKNOWLEDGEMENT}

This study was financially supported by the Department of Biotechnology, Ministry of Science and Technology of the Government of India. Authors would like to thank the Director of the Indian Veterinary Research Institute for providing the necessary facilities to carry out this work.

\section{REFERENCES}

Berndt, A. and U. Methner. 2001. Gamma/delta T cell response of chickens after oral administration of attenuated and nonattenuated Salmonella typhimurium strains. Vet. Immunol. Immunopathol. 78:143-161.

Cheeseman, J. H., M. G. Kaiser, C. Ciraci, P. Kaiser and S. J. Lamont. 2007. Breed effect on early cytokine mRNA expression in spleen and cecum of chickens with and without Salmonella enteritidis infection. Dev. Comp. Immunol. 31:5260.

Cheng, S. and S. J. Lamont. 1988. Breeding and genetic analysis of immunocompetence in White Leghorn chicken line. Poult. Sci. 67:989-995.

Dil, N. and M. A. Qureshi. 2002. Involvement of lipopolysaccharide related receptors and nuclear factor Kappa$\mathrm{B}$ in differential expression of inducible nitric oxide synthase in chicken macrophages from different genetic backgrounds. Vet. Immunol. Immunopathol. 88:149-161.

Djeraba, A., N. Bernarder, G. Dambrine and P. Quere. 2000. Nitric oxide inhibits Marek's disease virus replication but is not single decisive factor in interferon gamma mediated viral inhibition. Virology 277:223-234.

Djeraba, A., E. Musset, N. Bernarder, Y. L. Vern and P. Quere. 2002. Similar pattern of iNOS expression, NO production and cytokine response in genetic and vaccination acquired resistance to Marek's disease. Vet. Immunol. Immunopathol. 85:63-75.

Goldsby, R. A., T. J. Kindt and B. A. Osborne. 2003. Immunology. In: Freeman and Company (Ed. W. H. Freeman), New York.

Hangalapura, B. N., M. G. Kaiser, J. J. Poel, H. K. Parmentier and S. J. Lamont. 2006. Cold stress equally enhances in vivo proinflammatory cytokine gene expression in chicken lines divergently selected for antibody responses. Dev. Comp. Immunol. 30:503-511.

Harvey, W. R. 1975. Least squares analysis of data with unequal subclass frequencies. USDA Agric. Res. Services. ARS. H-4: 157.

Hussain, I. and M. A. Quereshi. 1997. Nitric oxide synthase activity and mRNA expression in chicken macrophages. Poult. Sci. 76:1524-1530.

Hussain, I. and M. A. Quereshi. 1998. The expression and regulation of inducible nitric oxide synthase gene differ in macrophages from chickens of different genetic background. Vet. Immunol. Immunopathol. 61:317-329.

Imanishi. 2004. Basis and clinical applications of interferon.
Journal of the Japan Medical Association 47:7-12.

Ahmed, K. A., V. K. Saxena, A. Ara, K. B. Singh, N. R. Sundaresan, M. Saxena and T. J. Rasool. 2007. Immune response to Newcastle disease virus in chicken lines divergently selected for cutaneous hypersensitivity. Int. J. Immunogenet. 34:445-455.

Kaiser, P., L. Rothwell, E. E. Galyov, P. A. Barrow, J. Burnside and P. Wigley. 2000. Differential cytokine expression in avian cells in response to invasion by Salmonella typhimurium, Salmonella enteritidis and Salmonella gallinarum. Microbiology 146:3217-3226.

Kogut, M., L. Rothwell and P. Kaiser. 2002. Differential effects of age on chicken heterophil functional activation by recombinant chicken interleukin-2. Dev. Comp. Immunol. 26:817-830.

Lamont, S. J. 1998. Impact of genetics on disease resistance. Poult. Sci. 77:1111-1118

Lillehoj, H. S. and G. Li. 2004. Nitric oxide production by macrophages stimulated with Coccidia sporozoites, lipopolysaccharide, or interferon-gamma, and its dynamic changes in SC and TK strains of chickens infected with Eimeria tenella. Avian. Dis. 48:244.

Lillehoj, H. S., W. Min and R. A. Dalloul. 2004. Recent progress on the cytokine regulation of intestinal immune responses to Eimeria. Poult. Sci. 83:611-623.

Macmicking, J., Q. W. Xie and C. Nathan. 1997. Nitric oxide and macrophage function. Annu. Rev. Immunol. 15:323-350.

Sadeyen, J. R., J. Trotereau, J. Protais, C. Beaumont, N. Sellier, G. Salvat, P. Velge and A. C. Lalmanach. 2006. Salmonella carrier-state in hens: study of host resistance by a gene expression approach. Microbes Infect. 8:1308-1314.

Sadeyen, J. R., J. Trotereau, P. Velge, J. Marly, C. Beaumont, P. A. Barrow, N. Bumstead and A. C. Lalmanach. 2004. Salmonella carrier state in chicken: comparison of expression of immune response genes between susceptible and resistant animals. Microbes Infect. 6:1278-1286.

Sarker, N., M. Tsudzuki, M. Nishibori, H. Yasue and Y. Yamamoto. 2000. Cell-mediated and humoral immunity and phagocytic ability in chicken lines divergently selected for serum immunoglobulin M and G levels. Poult. Sci. 79:1705-1709.

Sastry, K. V. H., R. P. Moudgal, J. Mohan, J. S. Tyagi and G. S. Rao. 2002. Spectrophotometric determination of serum nitrite and nitrate by copper-cadmium alloy. Anal. Biochem. 306:7982.

Sundaresan, N. R., K. A. Ahmed, V. K. Saxena, K. V. H. Sastry, M. Saxena, A. B. Pramod, M. Nath, K. B. Singh, T. J. Rasool, A. K. Devroy and R. V. Singh. 2005. Differential expression of inducible nitric oxide synthase and cytokine mRNA in chicken lines divergent for cutaneous hypersensitivity response. Vet. Immunol. Immunopathol. 108:373-385.

Swaggerty, C. L., M. H. Kogut, P. J. Ferro, L. Rothwell, I. Y. Pevzner and P. Kaiser. 2004. Differential cytokine mRNA expression in heterophils isolated from Salmonella-resistant and -susceptible chickens. Immunology 113:139-148.

Van Hemert, S., A. J. Hoekman, M. A. Smits and J. M. Rebel. 2007. Immunological and gene expression responses to a Salmonella infection in the chicken intestine. Vet. Res. 38:5163.

Wigley, P., S. Hulme, L. Rothwell, N. Bumstead, P. Kaiser and P. 
Barrow. 2006. Macrophages isolated from chickens genetically resistant or susceptible to systemic salmonellosis show magnitudinal and temporal differential expression of cytokines and chemokines following Salmonella enterica challenge. Infect. Immun. 74:1425-1430.
Withanage, G. S. K., P. Mastroeni, H. J. Brooks, D. J. Maskell and I. Mcconnell. 2005. Oxidative and nitrosative responses of the chicken macrophage cell line MQ-NCSU to experimental Salmonella infection. Br. Poult. Sci. 46:261-267.

Zhang-Barber, L., A. K. Turner and P. A. Barrow. 1999. Vaccination for control of Salmonella in poultry. Vaccine $17: 2538-2545$ 